\title{
MIP Models for the Hangar Space Utilization Problem with Safety Consideration
}

\author{
Yichen Qin ${ }^{1,2}$, Felix T.S. Chan ${ }^{2}$, S.H. Chung ${ }^{2}$, T. Qu ${ }^{1}$, X.P. Wang ${ }^{3}$, J.H. Ruan ${ }^{4,2}$ \\ ${ }^{1}$ School of Electrical and Information Engineering, Jinan University \\ Zhuhai 519070, China \\ yichen.qin@connect.polyu.hk; quting@jnu.edu.cn \\ ${ }^{2}$ Department of Industrial and Systems Engineering, the Hong Kong Polytechnic University \\ Hung Hom, Kowloon, Hong Kong, China \\ f.chan@polyu.edu.hk; nick.sh.chung@polyu.edu.hk \\ ${ }^{3}$ Faculty of Management and Economics, Dalian University of Technology \\ Dalian, China \\ wxp@dlut.edu.cn \\ ${ }^{4}$ College of Economics and Management, Northwest A\&F University \\ Yangling, China \\ junhu.ruan@polyu.edu.hk
}

\begin{abstract}
Maximizing the utilization of maintenance hangar is crucial for independent aircraft maintenance companies in order to meet the increasing maintenance demands from airline companies and survive in the intense competition within the industry. Hangar space is one of the resource constraints in aircraft maintenance scheduling problem. In this study, No-Fit Polygons (NFP) construction is adopted to prevent overlap between aircraft. Two Mixed-Integer Linear Programming (MILP) formulations are proposed with the objective to maximize the utilization of hangar while minimizing the risk of collision between aircraft. The proposed models are solved by the default branch-and-bound algorithm provided by CPLEX. Eight testing instances are generated based on the real-life data from an aircraft maintenance company. The computational results show that the default branch-and-bound algorithm is able to provide feasible parking layouts for all instances.
\end{abstract}

Keywords: aircraft maintenance, maintenance hangar, safety margin, mixed-integer programming, No-Fit Polygons

\section{Introduction}

Aircraft maintenance hangar utilization problem arises with the significant growth of maintenance demands from airline companies. Aircraft maintenance, repair and overhaul (MRO) activities are critical for aviation industry to ensure the operational safety and reliability of aircraft. Besides the maintenances checks called "line maintenance" that can be conducted at a gate or apron, the other checks that have to be conducted in hangar are categorized as "hangar maintenance". The cost of MRO activities becomes the third greatest cost center behind costs of fuel and operation, and maintenance cost represents around 9 percent of total annual operating cost for airlines [1] and maintenance activities cost more on airlines as the aircraft gets older with increasing maintenance requirements. Therefore, outsourcing maintenance activities to an independent aircraft maintenance company is an attractive alternative for airlines to ensure that their MRO operations continue to meet the safety requirements while maintaining minimum costs, maximizing quality and the best lead-time.

Fulfilling customers' needs within a reasonable time is a challenging task for maintenance company due to the increasing maintenance demands from different airline companies and limited hangar space and other maintenance resources cannot fulfill all of the demands at the same time. Therefore, aircraft from different customers are parked in the same hangar and maintenances are conducted concurrently to maximize the utilization of hangar in order to maximizes the overall profit during a period of time. Differ from the hangar maintenances operated by airline company, maintenance 
company receives demands from different airlines, which means that maintenance company need to arrange the aircraft in different sizes in the hangar together. Therefore, fixing parking stands, which is a common and widely adopted practice for airline-owned hangar, is not suitable for independent maintenance company since the parking plan and utilization of hangar differs based on the incoming customer orders. In addition, the safety consideration should be taken into account. The safety margin among aircraft should be maximized in order to reduce the risk of collision between aircraft as well as with staff and equipment in the maintenance processes.

\section{Literature Review}

A few literatures consider the line maintenance problems from aircraft maintenance company's perspective. Up to date, some researchers have studied integrated flight and maintenance planning problems in airline company to maximize overall profit. In addition, some literatures cover workforce scheduling problems from aircraft maintenance company's perspective. De Bruecker et al. [2] considered an aircraft maintenance personnel rosters problem in an independent aircraft line maintenance company, which assume that the maintenance routing problem is solved and the route are given for several airline companies. Liang et al. [3] considered an aircraft maintenance routing problem in the context to airline company. Up to date, Gavranis and Kozanidis [4] proposed an exact algorithm to maximize the fleet availability of a unit of military aircraft. Compared to the extensive literatures on scheduling problem in the context of airline company, the study on light and heavy maintenance in aircraft maintenance company is scarce.

There are some similarities between aircraft parking stands allocation problem and the fixed-dimension cutting and packing problem. Two-dimensional packing problem arises extensively in different industries whenever one needs to place multiple irregular items inside a container without overlap. According to Dyckhoff [5], those irregular items packing problems are referred as the nesting problem and mainly characterized by the number of relevant dimensions. Due to irregular shape of aircraft, the main difficulty of the problem is to ensure that the aircraft do not overlap with each other. In two-dimensional configuration, aircraft can be characterized as non-convex irregular polygons in Euclidian space. The most widely used tool for checking whether two polygons overlap is No-Fit Polygon (NFP). The main idea behind NFP are as follow: to find out whether two polygons overlap or not, the vector difference of the positions between the two polygons is calculated first, and then we conduct a simple test to identify whether the resulting vector difference is inside, outside or on the edge of NFP. Bennell and Oliveira [6] provide a detailed tutorial on how to generate NFP between two non-convex irregular polygons. Several integer programming formulations that solve nesting problem are proposed up to date. Major of them are proposed to solve the open dimension problem.

\section{Problem Description and Mathematical Formulation}

\subsection{Geometry Representation and Generation of NFPs}

The geometric shape of aircraft can be represented as polygon in two-dimensional Euclidian space (Fig. 1) and the middle point of the bottom of aircraft is denoted as reference point $\left(x_{i}, y_{i}\right)$.

Preventing overlap between aircraft is the primary consideration in parking stand allocation problem and we adopt the No-Fit Polygon construction to achieve this objective. Taking two polygons $p_{i}$ and $p_{j}$ as example, $N F P_{i j}$ is the region in with the reference point of $p_{j}$ cannot be placed if $p_{i}$ remain stationary since it would overlap aircraft $p_{i}$. The feasible zone for placing $p_{j}$ without overlap with $p_{i}$ is the region that outside $N F P_{i j}$. Given two polygons, the $N F P_{i j}$ is generated by tracing the path of the reference point on $p_{j}$ as $p_{j}$ slides around the boundary of $p_{i}$ such that two polygons always touch but never overlap (Fig. 2). Therefore, it is clear that if the reference point of $j$ moves into the $N F P_{i j}$ then two polygons overlap, therefore the interior of the $N F P_{i j}$ represents all overlapping positions. The two polygons touch when the reference point of $p_{j}$ is on the boundary of the $N F P_{i j}$, and they separate when the reference point is outside the $N F P_{i j}$. 


\subsection{Partitioning feasible region outside NFPs and Concept of Slices}

Alvarez-Valdes et al. [7] improved the approaches of Fischetti and Luzzi [8] by partitioning the NFP to several horizontal slices (as shown in Fig. 2) in order to formulate mixed integer programming models. Horizontal slices are defined by drawing one horizontal line outward from each vertex and then each slice is characterized by one or two horizontal edge(s) and a segment of boundary of NFP (Fig. 3). A set of variables $b_{i j k}$ are associated to each horizontal slice and the reference point of $p_{j}$ is placed in the slice $k$ if $b_{i j k}=1$. Therefore, a general form of the constraint preventing overlapping is $\quad \alpha_{i j}^{k f}\left(x_{j}-x_{i}\right)+\beta_{i j}^{k f}\left(x_{j}-x_{i}\right) \leq q_{i j k}+M \cdot\left(1-b_{i j k}\right), \forall i, j \in P, i \neq j, k=1,2, \ldots, m_{i j} \quad$ where $\alpha_{i j}^{k f}\left(x_{j}-x_{i}\right)+\beta_{i j}^{k f}\left(x_{j}-x_{i}\right)=q_{i j k}$ is the equation of the line including the $k$ th edge of $N F P_{i j} . m_{i j}$ is the total number of slices of $N F P_{i j}$. In open dimension nesting problem [7,8], we enforce $\sum_{k=1}^{m_{i j}} b_{i j k}=1$ since all items are placed in the container with infinite length.

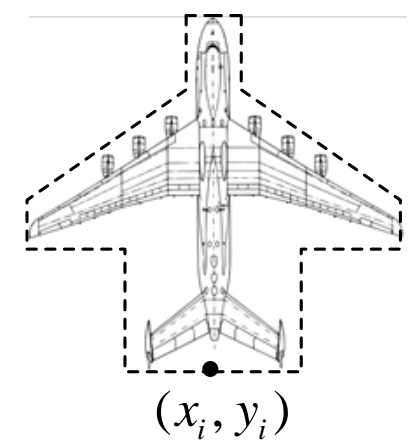

Fig. 1: Geometric Representation and Reference Point of Aircraft in two-dimensional space.

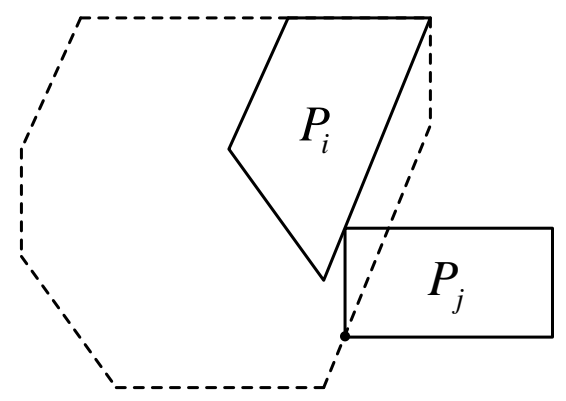

Fig. 2: No-fit polygon $N F P_{i j}$.

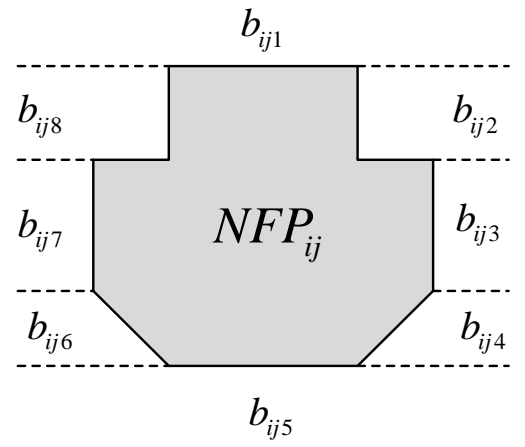

Fig. 3: Horizontal slices outside NFP. 


\subsection{Model Formulation}

\subsubsection{Phase I Problem}

Given a number of maintenance orders that the given hangar cannot accommodate all of them concurrently, we aim to find out the subset of maintenance orders with maximal utilization and a feasible parking plan as well. The problem is equivalent to minimize the wasted space of the hangar space.

\section{Notations}

$W$

$H$

$i$

$P$

$A_{u}$

$v_{i}$

$w_{i}$

$h_{i}$

$$
\alpha_{i j}^{k f}, \beta_{i j}^{k f}, q_{i j}^{k f}
$$

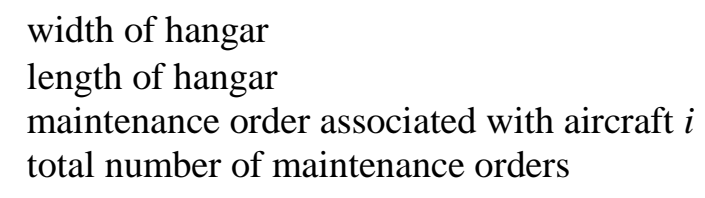

width of hangar

length of hangar

maintenance order associated with aircraft $i$

total number of maintenance orders

$m_{i j}$

a group of aircraft that belong to the same model $u$

area of aircraft $i$

width of aircraft $i$

length of aircraft $i$

$M$ large number

Decision Variables

$z_{i} \quad 1$, if aircraft $i$ is placed in hangar

$x_{i}$

position of reference point of aircraft $i$ on $x$-axis in two-dimensional space

$y_{i}$ position of reference point of aircraft $i$ on y-axis in two-dimensional space

$b_{i j k}$ 1 , if the reference point of aircraft $j$ is placed into the slice $s_{i j}^{k}$ when the aircraft $i$ remains stationary

$$
\begin{gathered}
\text { Objective } \text { : Maximize Hangar Utilization } \\
\text { Min HangarArea }-\sum_{i=1}^{p} v_{i} z_{i} \\
\text { s.t. } x_{i}+w_{i} / 2 \leq W, \forall i \in P \\
x_{i} \geq w_{i} / 2-M \cdot\left(1-z_{i}\right), \forall i \in P \\
y_{i}+h_{i} \leq H, \forall i \in P \\
\alpha_{i j}^{k f}\left(x_{j}-x_{i}\right)+\beta_{i j}^{k f}\left(x_{j}-x_{i}\right) \leq q_{i j}^{k f}+M \cdot\left(1-b_{i j k}\right), \forall i, j \in P, i \neq j, \forall k=1,2, \ldots, m_{i j}, \forall f=1,2, \ldots, t_{i j}^{k} \\
\sum_{k=1}^{m_{i j}} b_{i j k} \leq z_{i}, \forall i, j \in P, i \neq j, k=1,2, \ldots, m_{i j} \\
\sum_{k=1}^{m_{i j}} b_{i j k} \leq z_{j}, \forall i, j \in P, i \neq j, k=1,2, \ldots, m_{i j} \\
\sum_{k=1}^{m_{i j}} b_{i j k} \geq z_{i}+z_{j}-1, \forall i, j \in P, i \neq j, k=1,2, \ldots, m_{i j}
\end{gathered}
$$




$$
\begin{gathered}
b_{i j k} \in\{0,1\} \forall i, j \in P, i \neq j, k=1,2, \ldots, m_{i j} \\
z_{i} \in\{0,1\} \quad \forall i \in P
\end{gathered}
$$

In this formulation, the objective function (1) minimizes the wasted space in maintenance hangar, which is equivalent to maximize the utilization of hangar. Constraints (2) - (4) are bound constraints that ensure aircraft do not exceed the hangar boundary. Constraint (5) prevents overlap between each pair of aircraft in hangar. Variables $b_{i j k}$ are binary variables and one of them must take value 1 if aircraft $i$ and $j$ are placed in hangar (Constraints (6) - (8)). For those aircraft that is to be placed in hangar, we set $z_{i}=1$ that activate constraint (4) - (8) to impose non-overlapping constraints for every aircraft in hangar, otherwise, $z_{i}=0$ deactivates non-overlapping constraints for those aircraft that is not going to be placed in hangar.

\subsubsection{Phase II Problem}

After the subset of maintenance orders with maximal utilization is found, the objective in Phase II is to maximize the overall safety margins in the existing parking plan by enlarging the safety margin between each pair of aircraft. The definition of safety margin is the shortest distance between two aircraft in Euclidian space (Fig. 4) and the overall safety margin is calculated based on the sum of shortest distance of each aircraft placed in the hangar. Since the large-sized aircraft bear more risk of collision with the other aircraft, the weight of each aircraft in the objective function is associated with their respective aircraft area.

To enlarge and ensure the safety margin between two aircraft, the approach is to use the property and the definition of $N F P$. According to the definition of $N F P$, polygon $j$ touches polygon $i$ if the reference point of polygon $j$ is placed on any edge of NFP. In order to enlarge the shortest distance from "touch" each other to a "safety distance", we simply add a "buffer area" to NFP by moving the edges of original NFP outward (Fig. 4), then the shortest distance between two aircraft can therefore been guaranteed from any direction. The primal decision variables in this phase are safety margin of each aircraft.

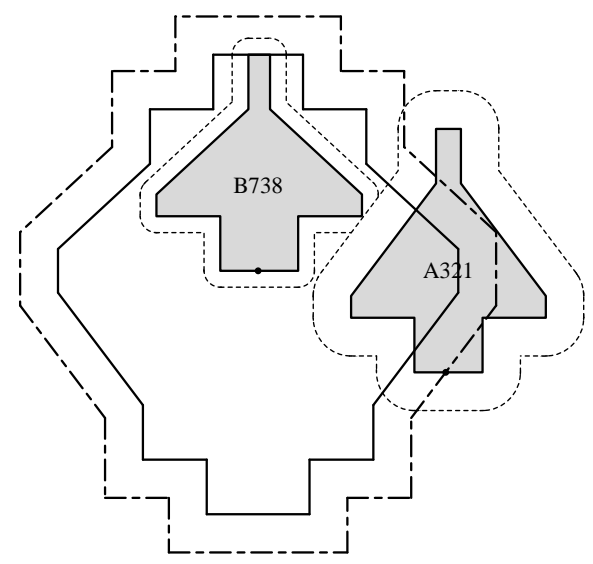

Fig. 4: Revised NFP and Safety Margin between Aircraft.

Some new notations and decision variables are introduced for the formulation of Phase II problem:

\section{Notations}

$N F P_{i j}^{n}$ the revised NFP between aircraft $i$ and $j$ with safety margin $n$

$\alpha_{i j n}^{k f}, \beta_{i j n}^{k f}, q_{i j n}^{k f}$ parameters used to define the $f$ th linear inequality of the kth slice outside $N F P_{i j}^{n}$

$m_{i j}^{n}$ number of slices outside $N F P_{i j}^{n}$ 
$t_{i j n}^{k} \quad$ number of linear inequalities of the $k$ th slice outside $N F P_{i j}^{n}$

ubper bound of safety margin

lb lower bound of safety margin

Area $_{i} \quad$ area of aircraft $i$

Decision Variables

$z_{i}^{n}$

1 , if aircraft $i$ is placed in hangar with safety margin $n$

$b_{i j k}^{n} \quad 1$, if the reference point of aircraft $j$ is placed into $s_{i j n}^{k}$ while the aircraft $i$ remains stationary

$g_{i j}^{n} \quad 1$, if $N F P_{i j}^{n}$ is activated to separate two aircraft and the safety margin between these two aircraft is $n$

$$
\begin{aligned}
& \text { Objective: Maximize Overall Safety Margins } \\
& \operatorname{Max}_{i}^{P^{\prime}} \cdot \sum_{n=l b}^{u b} z_{i}^{n} \cdot n \cdot \text { Area }_{i} \\
& \text { s.t. } x_{i}+w_{i} / 2 \leq W, \forall i \in P^{\prime} \\
& x_{i} \geq w_{i} / 2, \forall i \in P^{\prime} \\
& y_{i}+h_{i} \leq H, \forall i \in P^{\prime} \\
& \alpha_{i j n}^{k f}\left(x_{j}-x_{i}\right)+\beta_{i j n}^{k f}\left(x_{j}-x_{i}\right) \leq q_{i j n}^{k f}+M \cdot\left(1-b_{i j k}^{n}\right), \forall i, j \in P^{\prime}, i \neq j, \forall k=1,2, \ldots, m_{i j n}, \forall f=1,2, \ldots, t_{i j n}^{k}, n=l b, \cdots, u b \\
& \sum_{k=1}^{m_{i n n}} b_{i j k}^{n}=g_{i j}^{n}, \forall i, j \in P^{\prime}, i \neq j, n=l b, \cdots, u b \\
& g_{i j}^{n} \geq z_{i}^{n}-\sum_{n=n+1}^{u b} z_{j}^{n}, \forall i, j \in P^{\prime}, i \neq j \\
& g_{i j}^{n} \geq z_{j}^{n}-\sum_{n=n+1}^{u b} z_{i}^{n}, \forall i, j \in P^{\prime}, i \neq j \\
& g_{i j}^{n} \leq 1-\sum_{n=n+1}^{u b} z_{i}^{n}, \forall i, j \in P^{\prime}, i \neq j \\
& g_{i j}^{n} \leq 1-\sum_{n=n+1}^{u b} z_{j}^{n}, \forall i, j \in P^{\prime}, i \neq j \\
& \sum_{n=l b}^{u b} g_{i j}^{n}=1, \forall i, j \in P^{\prime}, i \neq j \\
& \sum_{n=l b}^{u b} z_{i}^{n}=1, \forall i \in P^{\prime} \\
& b_{i j k}^{n} \in\{0,1\} \forall i, j \in P^{\prime}, i \neq j, k=1,2, \ldots, m_{i j n}, \\
& g_{i j}^{n} \in\{0,1\} \quad \forall i, j \in P^{\prime}, i \neq j, \forall n=l b, \cdots u b
\end{aligned}
$$

In (F2) formulation, the objective function (11) maximizes the overall safety margins, which is measured by the sum of aircraft area times its individual safety margin for each aircraft in hangar. Constraints (12) - (14) are bound constraints that keep aircraft in hangar. Constraint (15) separates each pair of aircraft by the safety margin $n$ if the 
constraints are activated. Sets of $b_{i j k}^{n}$ are binary variables and one of them must take value 1 if aircraft $i$ and $j$ are placed in hangar and $g_{i j}^{n}=1$, which indicates the safety margin of this pair of aircraft is $n$ (Constraints (16)). Set of $z_{i}^{n}$ for each aircraft indicate the safety margin of aircraft $i$ and one of them must take value one for each aircraft (Constraint (22)). Since the safety distance between two aircraft is determined by the aircraft with larger safety margin in that pair, Constraints (17) - (21) ensure that $g_{i j}^{n}$ is determined by the larger safety margin in the pair of aircraft $i$ and $j$.

\section{Computational Experiments and Results}

\subsection{Test Settings}

The results of the computational experiments are presented in this section. All the procedures described in the previous sections are coded in C\# in Visual Studio 2010 and run on a computer with an Intel Core i7 processor, at $3.6 \mathrm{GHz}$ with 64 $\mathrm{Gb}$ of RAM. The mixed-integer linear programming is solved by the CPLEX 12.5 serial model.

The instances are created based on real-life data from an aircraft maintenance company located in Hong Kong. We generate the testing instances with maintenance orders ranged from 6 to 12 aircraft for each instance. The dimension of hangar is fixed at 110 meters long and 110 meters wide. The entire set is divided into 4 groups of 8 instances while the proportions of aircraft in each group of instances are different. In particular, 8 instances are divided into four groups: 1) the majority of aircraft are small-sized, 2) the majority of aircraft are medium-sized, 3) the majority of aircraft are large-sized, and 4) the number of aircraft from different categories is equal.

\subsection{Computational results}

Tables 1 and 2 show the computational results for 8 instances in Phase I and II problem. In Table 1, the first column stands for instance name and the "S/M/L" in the second column stands for number of aircraft from three categories, respectively. The number of binary variables involved in each instances is reported in the third column. The best objective incumbent values (lower bound) in Phase I problem are indicated in the fourth column. The column ('Time') and ('Gap') in the fifth and sixth column report the CPU time elapsed when termination criteria was met as well as the relative gap, respectively. The seventh column represents the number of aircraft placed in hangar after computation. Similarly, Table 2 reports the computational results for the Phase II problem. The time limit for each instance is 3600 seconds for the Phase I problem, and 18000 seconds for the Phase II problem and the upper bound of safety margin is 8 meters in Phase II problem. In Phase I problem, all the original NFPs are revised by safety margin in one meter as the minimum safety margin between each pair of aircraft.

Table 1: Computational Results for the Phase I Problem.

\begin{tabular}{|c|c|c|c|c|c|c|}
\hline \multirow{2}{*}{$\begin{array}{l}\text { Instanc } \\
\mathrm{e}\end{array}$} & \multirow{2}{*}{$\begin{array}{l}\text { Aircraft } \\
\text { S/M/L }\end{array}$} & \multirow{2}{*}{$\begin{array}{l}\text { Number of binary } \\
\text { variables in Phase I }\end{array}$} & \multicolumn{4}{|c|}{ Phase I } \\
\hline & & & LB & Time & Gap & Number of aircraft in hangar \\
\hline 1 & $4 / 2 / 2$ & 738 & 6770.92 & 3.69 & 0 & 8 \\
\hline 2 & $7 / 1 / 1$ & 993 & 7884.61 & 2.95 & 0 & 9 \\
\hline 3 & $3 / 7 / 0$ & 1192 & 8717.18 & 3.52 & 0 & 10 \\
\hline 4 & $1 / 5 / 2$ & 734 & 5965.05 & 5.75 & 0 & 8 \\
\hline 5 & $2 / 1 / 4$ & 587 & 6253.1 & 35.68 & 0 & 6 \\
\hline 6 & $3 / 2 / 5$ & 1234 & 5698.1 & 250.39 & 0 & 8 \\
\hline 7 & $3 / 3 / 3$ & 961 & 5670.51 & 5.06 & 0 & 9 \\
\hline 8 & $4 / 4 / 4$ & 1776 & 4669.42 & 3600 & 36.49 & 10 \\
\hline
\end{tabular}


Table 2: Computational Results for the Phase II Problem.

\begin{tabular}{|l|l|l|l|l|l|}
\hline Instance & Number of binary & \multicolumn{3}{|c|}{ Phase II } \\
\cline { 3 - 6 } & variables in Phase & \multicolumn{1}{|c|}{ LB } & \multicolumn{2}{c|}{ TB } & \multicolumn{1}{c|}{ Gap } \\
\hline 1 & 5126 & & & 18000 & 13.12 \\
\hline 2 & 6888 & 36033.94 & 40762.74 & 17.68 & 0 \\
\hline 3 & 8286 & 33723.12 & 33712.12 & 134.57 & 0 \\
\hline 4 & 5128 & 27062.56 & 27062.56 & 18000 & 220.94 \\
\hline 5 & 2930 & 12629.45 & 40533.24 & 198.03 & 0 \\
\hline 6 & 5262 & 18560.56 & 18560.56 & 18000 & 86.51 \\
\hline 7 & 6704 & 19526.52 & 36418.47 & 18000 & N/A \\
\hline 8 & 8312 & N/A & 51435.92 & 18000 & N/A \\
\hline
\end{tabular}

The results show that the default branch-and-bound algorithm by CPLEX is able to find the feasible solution for all instance in Phase I problem and most of them are solved to optimal. However, as the problem size grows, the efficiency of the algorithm deteriorates especially for the Phase II problem since high numbers of binary variables are involved to determine the relative position between every pair of aircraft as well as the safety margin of each aircraft. Advanced approaches shall be developed to tighten the bounds of formulation to improve the computational efficiency of the exact algorithm. The layouts of hangar in 8 instances are shown in Fig. 5.

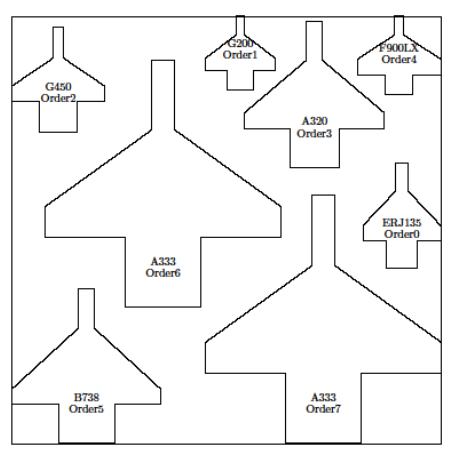

1

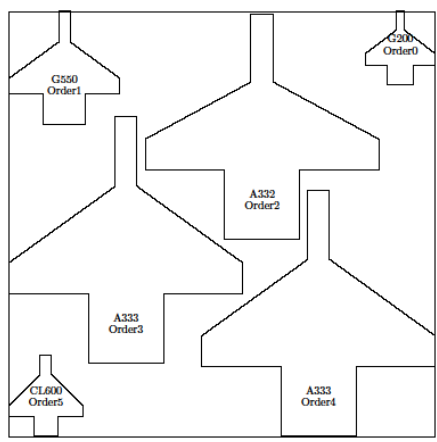

5
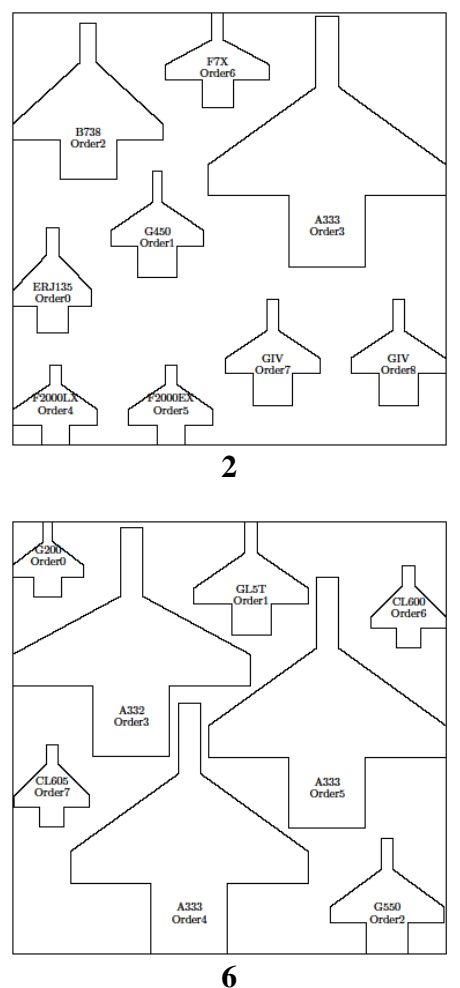

Fig. 5: Layouts of hangar in 8 instances.

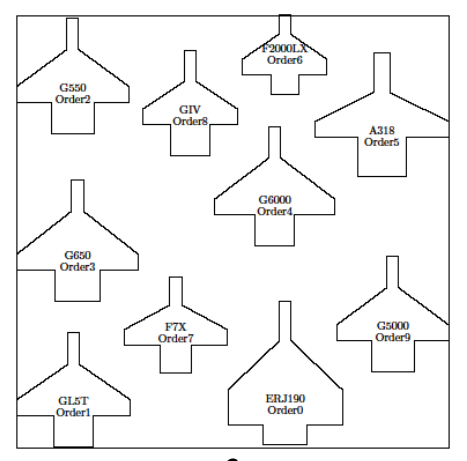

3

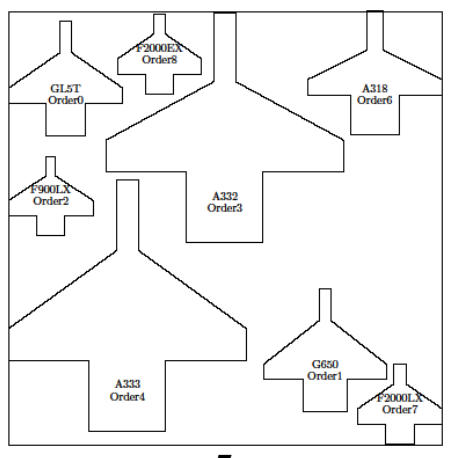

7

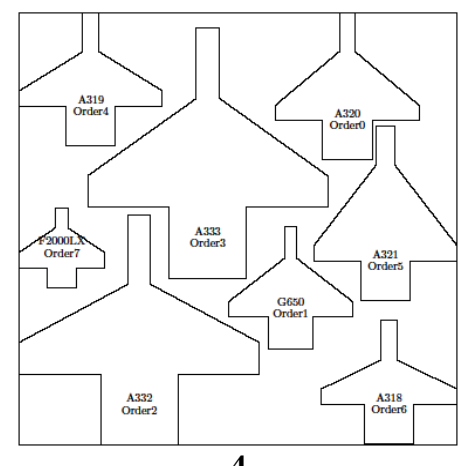

4

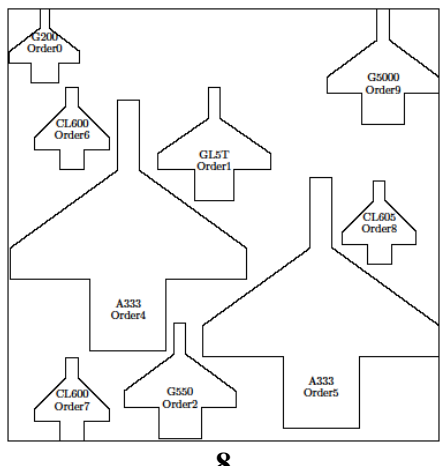

8

\section{Conclusion and Future Work}

This paper describes two mixed-integer programming formulations for the utilization of hangar space in an aircraft maintenance company, which is a critical problem in the operation of maintenance activities and seldom considered in the literature. The problem is formulated and solved in two stages to maximize the utilization as well as the overall safety margins. The computational results show that the default branch-and-bound algorithm provided by CPLEX is able to solve most of the small instances. 
We expect that these preliminary results can be further improved by proposing advanced branching strategies given the hierarchal structure of binary variables as well as adding valid inequalities in the original problem to tighten the formulation. Moreover, heuristic can also be implemented to obtain a better initial solution in the Phase II problem.

\section{Acknowledgements}

The work described in this paper was supported by grants from the Research Grants Council of the Hong Kong Special Administrative Region, China (Project No. PolyU 15201414); The Natural Science Foundation of China (Grant No. 71471158); The Hong Kong Polytechnic University Research Committee (Project Numbers G-UB03, G-YBFD, GUA4F); and student account code RUF1.

\section{References}

[1] ITAT, Airline Maintenance Cost Executive Commentary, [Online]. Available: https://www.iata.org/whatwedo/workgroups/Documents/MCTF/AMC-Exec-Comment-FY14.pdf

[2] P. De Bruecker, J. Van den Bergh, J. Belien, E. Demeulemeester, "A model enhancement heuristic for building robust aircraft maintenance personnel rosters with stochastic constraints," European Journal of Operational Research, vol. 246, no. 2, pp. 661-673, 2015.

[3] Z. Liang, Y. Feng, X. N. Zhang, T. Wu, W. A. Chaovalitwongse, "Robust weekly aircraft maintenance routing problem and the extension to the tail assignment problem," Transportation Research Part B-Methodological, vol. 78, pp. 238-259, 2015.

[4] A. Gavranis, G. Kozanidis, "An exact solution algorithm for maximizing the fleet availability of a unit of aircraft subject to flight and maintenance requirements," European Journal of Operational Research, vol. 242, no. 2, pp. 631-643, 2015.

[5] H. Dyckhoff, "A Typology of Cutting and Packing Problems," European Journal of Operational Research, vol. 44, no. 2, pp. 145-159, 1990.

[6] J. A. Bennell, J. F. Oliveira, "A tutorial in irregular shape packing problems," Journal of the Operational Research Society, vol. 60, no. S93-S105, 2009.

[7] R. Alvarez-Valdes, A. Martinez, J. M. Tamarit, "A branch \& bound algorithm for cutting and packing irregularly shaped pieces," International Journal of Production Economics, vol. 145, no. 2, pp. 463-477, 2013.

[8] M. Fischetti, I. Luzzi, "Mixed-integer programming models for nesting problems," Journal of Heuristics, vol. 15, no. 3, pp. 201-226, 2009. 